\title{
The Fungal to Allergen Index (FAI): A new concept to detect mould problems in buildings
}

\author{
Morten Reeslev ${ }^{\mathrm{a}, *}$ \\ ${ }^{a}$ Mycometer A/S Horsholm, Denmark \\ mreeslev@mycometer.com
}

\begin{abstract}
The Fungal to Allergen Index is a new concept aiming at giving better information of the Indoor Air Quality. Parallel air samples are analysed for total fungi and total allergens. This gives information of the potential exposure to fungi and allergens but also an indication of the risk of a mould source. Investigations of "non-problem buildings" having no apparent moisture or mould problems, and a few buildings with mould/moisture problems showed that the median value was $8.5 \%$ in non-problem buildings and all values was above $56 \%$ in buildings with mould/moisture problems.

Peer-review under the responsibility of the organizing committee of the ICMB21.
\end{abstract}

Keywords: Fungi/Mould; Activated air sampling; Allergens; Reproducibility; Fungal to Allergen Index

\section{Introduction/Background}

Quantification of fungal spores by cultivation or microscopy is still the main approach for supporting the visual assessment of buildings for mould problems. Comparing the total level of spores and the rank, order and diversity of fungal genera in indoor and outdoor samples are used to predict whether or not the indoor environmental conditions are different from the outdoor and therefore indicating a mould problem as defined by the presence of fungal growth. This approach is slow in taking days before a result can be obtained. Also few laboratories can consistently correctly determine the fungi to species level. In the present study we present a whole new concept the Fungal to Allergen Index (FAI). The results can be obtained in a few hours and independent of subjective evaluations from the personnel involved.

Two parallel air samples using activated sampling are taken. One sample is analysed for the presence of total allergens, and measures the potential exposure for allergens. The other sample is analysed for the level of fungi, and measures the potential exposure for fungi. The FAI principle is when the two results are combined and used to predict the likelihood of the presence of a source of fungi in or in the vicinity of the room.

\section{Methods}

Activated air sampling was performed using a Makita BUB182Z cordless leaf blower (Flow rate $2.6 \mathrm{~m} 3 / \mathrm{min}$.) to blow across surfaces, starting with the ceiling and working the way down ending with to the floor. The blowing was performed keeping a distance of approximately 2 meters to from all surfaces and using approximately 1 min per $10 \mathrm{~m} 2$ room. The sampling time was 10 minutes using a flow rate of $15 \mathrm{lpm}$. The level of fungi and the level of total allergens were measured by determining the level of a specific fungal enzyme activity and an enzyme activity present in allergenic material present in buildings (dust mites, dander, pollen, fungi insects etc.). The enzyme reactions occur directly on each respective filter after which the fluorescence formed during the enzyme reaction, are determined. Results are given in fluorescence units (FLU).

The FAI is calculated as: $F A I=\frac{\text { Mould level }(F L U)}{\text { Total allergens }(F L U)} \times 100 \%$

\section{Results}

Measurement of total fungi and total allergens in 250 air samples were performed in buildings with no water or mould problems as well as 11 samples from buildings with mould problems.

The main result of the study was that, the FAI were generally much lower in buildings with no mould or water problems. In the 'non-problem buildings', the median value was around $8 \%$, meaning $8 \%$ of the total allergen signal was of fungal origin. In the buildings with mould/moisture problems, all samples had a FAI between $56 \%$ and $100 \%$.

\section{Conclusions}

The FAI concept consists of a rapid assessment of the of the potential exposure level of both fungi and of total allergens in the indoor environment and also the risk of the presence of a mould source. More data is being collected in problem and nonproblem buildings to further define and refine interpretation criteria. 